\title{
Anthraquinone Grafted Graphene Oxide/Polypyrrole Composites with Enhanced Elec- trochemical Performance
}

\author{
Yikun Wang, Yongqin Han", Tingxi Li, Zonglin Zhang, Zhongxing Chen, Aiping Cao and Yanmin Wang \\ Department of Polymer Material, College of Materials Science and Engineering, Shandong University of Science and Technology, \\ Qingdao 266510, PR China
}

Corresponding Author Email: yongqinhan@126.com

\begin{abstract}
1,4- diaminoanthraquinone (DAQ) grafted graphene oxide (DGO) is prepared by the ring opening reaction of epoxy group on GO surface with $\mathrm{NH}_{2}$ in DAQ. DAQ-GO/PPy composites (DGPS) have been prepared by a facile chemical oxidative polymerization method with different DGO weight loadings (3wt\%, 5wt\%, 9wt\% and 15wt\%). The introduction of redox active DGO thus endows DGPs with improved electrochemical performances. Among the DGPs, DGP5 provides the best electrochemical performances due to the delaminated DGO sheets as well as interconnect composite microstructure, which is desirable for superior charge storage. The specific capacitance of DGP5 can reach $389 \mathrm{~F} \mathrm{~g}^{-1}$, which is more than two times as much as that of pristine PPy. The high specific capacitance, enlarged window (as much as $1.4 \mathrm{~V}$ ) as well as the good cycle stability, which are ascribed to the synergistic effect as well as the redox activity of DGO could be benefit for DGP5 as promising supercapacitor electrodes.
\end{abstract}

Keywords: graphene oxide; polypyrrole; redox active; composites; supercapacitor

Received: July-04-2018, Accepted: February-05-2019, https://doi.org/10.14447/jnmes.v22i4.a03

\section{INTRODUCTION}

Nowadays, global demand for environmentally friendly high performance energy conversion and storage is increasing rapidly due to the population and economic growth as well as the higher consumption of fossil fuels. Supercapacitors (also called ultracapacitors) are environmental-friendly high capacity capacitors bridging the gap between ordinary capacitors and batteries. They can provide high power capacity, fast charge-discharge rates and long cycle life[1]. According to the energy storage mechanism, supercapacitors can be commonly divided into two categories. One is electrochemical double-layer capacitors, the electrode materials of which are usually carbon materials (such as graphene [2-4], carbon nanotube [5,6], active carbon [7,8], etc.) that store charge through rapid ion adsorption/desorption process at the electrode/electrolyte. The other is pseudocapacitors that employ conductive polymers and transition metal oxides/hydroxides whose high capacitance and energy density originated from the surface or nearsurface reactions of electroactive species[9-13].

Among the carbon materials, graphene with 2D lamellar structure used as supercapacitor electrode materials has attracted attention due to its remarkable high electrical conductivity, ultra-large theoretical specific surface area $\left(2630 \mathrm{~m}^{2} \mathrm{~g}^{-1}\right)$, high mechanical strength as well as superior chemical/electrochemical stability [14-17]. However, the specific surface area of bulk graphene cannot meet that in theory because the intersheet van der Waals attractions and $\Pi-\pi$ stacking interactions might lead to the re-aggregation of graphene sheets [14-17]. Introducing the conductive polymers into graphene can reduce the reaggregation of graphene sheets. Owing to the low-cost, easy synthesis, reversible redox and doping/dedoping reactions, polypyrrole (PPy) is considered as one of the most promising electrode materials for supercapacitors [21-24]. Unfortunately PPy usually suffers from poor cycle stability due to continuous swelling/shrinkage of polymer chains oc- curring during the charge/discharge (doping/dedoping) processes. The applications of PPy used as supercapcitor electrode materials thus have been limited $[25,26]$.

In order to update the properties of both graphene- and PPy-based supercapacitors, very recently researchers have attempted to prepare binary graphene-PPy composites or even ternary composites as supercapacitor electrode active materials to improve electrochemical performance of supercapacitors via synergistic effects [27-32]. The reported composites can provide high specific capacitances and improved cycle stabilities. The ultimate supercapacitor performances (i.e., specific capacitance, cycling stability, charge-discharge properties, etc.) can be determined by various factors such as polymerization method (chemical oxidative polymerization, electrochemical polymerization), intrinsic properties of PPy electrolyte, dispersion/aggregation of the graphene in the composites, different properties of graphene used, etc. Different PPy microstructures, such as "cauliflower" or nanosheet morphology [27], spherical nanoparticles [28], nanorods [29], etc. and the constructed composite porous microstructure are also obtained, providing unobstructed pathways for the fast diffusion and exchange of ions/electrons. However, although compared with pure PPy and graphene, the electrochemical performances of graphene/PPy nanocomposites have been improved greatly, the energy density and power density of the composites are still lower than lithium-ion batteries owning to the limitation of charge accumulation ability [33], thus leading to the supercapacitors unable to meet practical application requirements. The introduction of redox active molecules can further improve the electrochemical properties graphene/PPy based supercapacitors, especially the specific capacitance and potential window.

In this work, 1,4-diaminoanthraquinone (DAQ), which can improve the electrochemical activity through the redox reaction of quinone/hydroquinone, is grafted onto graphene oxide (GO) surface and the DAQ grafted GO (DGO) is obtained. The DAQ thus endowed GO 

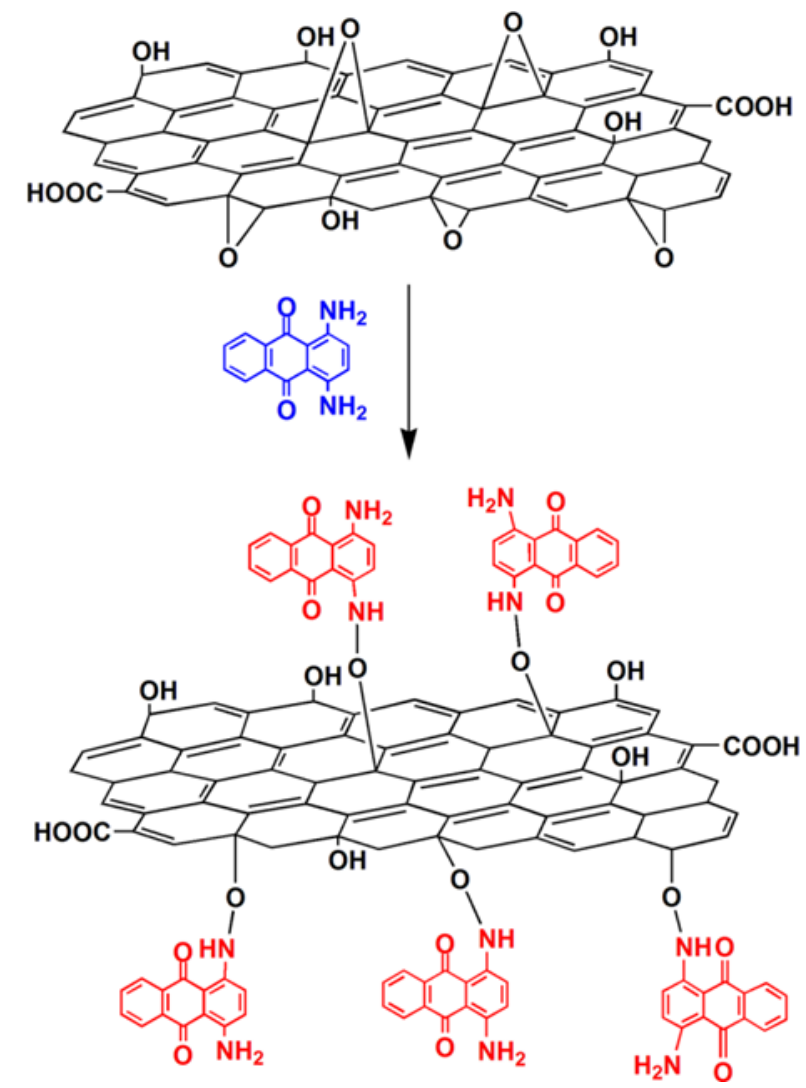

Figure 1. The schematic diagram of the Grafting procedure of DAQ

with redox activity and different ratios of DAQ-GO/PPy composites (DGPs) have been prepared. The functional groups on DGO can also induce ordered polymerization of PPy. By adjusting the weight ratio of DGO to PPy, the stacking of both PPy particles and the graphene sheets can be hindered, which is benefit for the improvement of the electrochemical performances.

\section{EXPERIMENTAL}

\subsection{Synthesis of DAQ covalently grafted GO (DGO)}

The typical synthesis procedure are as follows: $1 \mathrm{~g}$ DAQ was dissolved in $100 \mathrm{~mL}$ ethanol with magnetic stirring for $30 \mathrm{~min}$ and sonication for $1 \mathrm{~h} .0 .1 \mathrm{~g}$ GO was added to $100 \mathrm{ml}$ ethanol with magnetic stirring $30 \mathrm{~min}$ to make it dispersed completely. The above two kinds of solution were mixed with constant stirring at $90{ }^{\circ} \mathrm{C}$ for $30 \mathrm{~h}$. The collected powder was purified with deionized water and ethanol. The powder was dissolved in deionized water, sonicated for 2 hours and filtered to prepare DGO solution. The reaction scheme of the DGO is illustrated in Figure1.

\subsection{Preparation of DGO/ PPy Composites (DGPs)}

The DGO loadings are various from $3 \mathrm{wt} \%, 5 \mathrm{wt} \%, 9 \mathrm{wt} \%$ to $15 \mathrm{wt} \%$ and the resulting composites are named as DGP3, DGP5, DGP9 and DGP15, respectively.

Taking the DGP3 as an example. $70 \mu 1$ Py $(1 \mathrm{mmol})$ was injected to $7 \mathrm{~mL}$ DGO solutions (concentration: $0.3 \mathrm{mg} / \mathrm{mL}$ ) with magnetic stirring for $12 \mathrm{~h}$. Then $0.2282 \mathrm{~g}$ ammonium persulfate (APS) $(1 \mathrm{mmol}$, dissolved in $5 \mathrm{~mL}$ of deionized water) and $0.25 \mathrm{mmol}$ fumaric acid (used as dopant of PPy) were added to the above solution. The reaction was allowed to proceed for $12 \mathrm{~h}$ at room temperature. After purification

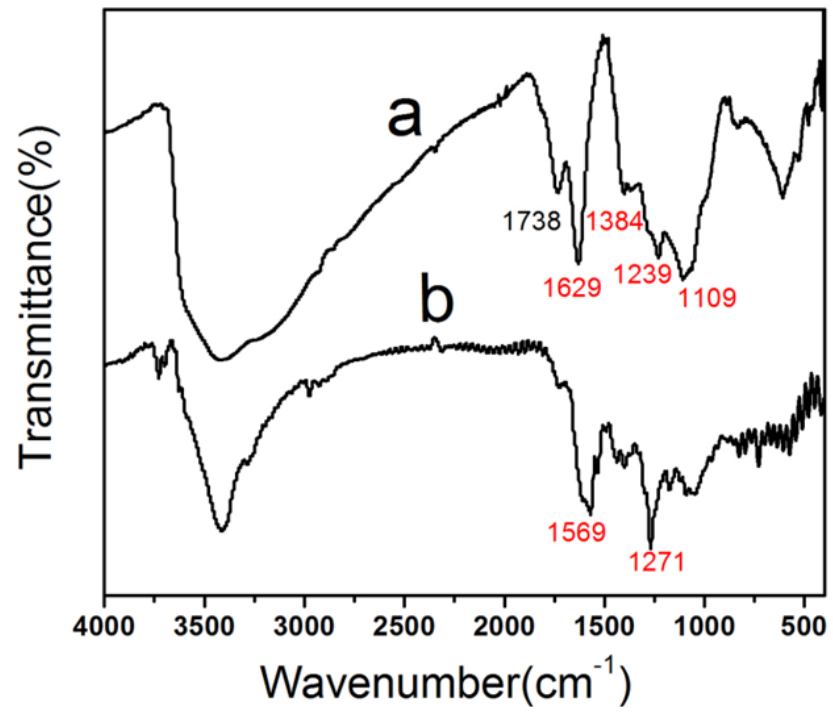

Figure 2. The FTIR spectra of (a) GO and (b) DGO

with deionized water and ethanol, the obtained product was dried in a vacuum oven for $24 \mathrm{~h}$ at $60{ }^{\circ} \mathrm{C}$.

As a comparison, pure PPy was prepared with APS as oxidant.

\subsection{Material characterization}

Scanning electron microscopy (SEM) measurements were carried out by a Nova Nano SEM 450 scanning electron microscope. The specimens were platinum-coated prior to measurement. A Nicolet 380 FT-IR spectrometer was used to record the FTIR spectra of the obtained samples using pressed $\mathrm{KBr}$ pellets. X-ray diffraction (XRD) patterns were recorded on a D/MAX-2500PCX X-ray diffractometer with $\mathrm{Cu} \mathrm{K} \alpha$ radiation $\mathrm{n}(\lambda=0.1541 \mathrm{~nm})$.

\subsection{Electrochemical measurements}

The electrochemical performances are investigated by a threeelectrode system. The working electrode is prepared by firstly mixing the electroactive materials, carbon black and polyterafluoroethylene (PTFE) (mass ratio of 85:10:5 ) homogeneously and then pressed on graphite current collector. The counter and reference electrodes used are platinum foils and saturated calomel electrode (SCE), respectively. $1 \mathrm{M} \mathrm{H}_{2} \mathrm{SO}_{4}$ was used as electrolyte. Cyclic voltammetry (CV) and electrochemical impedance spectroscopy (EIS) were carried out on Parstat 2273 electrochemical workstation. CV tests were performed between 0.9 and $0.5 \mathrm{~V}$ at $10 \mathrm{mV} \mathrm{s}^{-1}$. Galvanostatic charge/discharge (GCD) and cycling measurements were carried out between -0.8 and $0.2 \mathrm{~V}$ with a LAND cell testing system (Szland CT2001A). EIS measurements were conducted at open circuit potential in the frequency range from $10^{5}$ to $0.01 \mathrm{~Hz}$ with an ac perturbation of $5 \mathrm{mV}$.

\section{RESULTS AND DISCUSSION}

\subsection{Structure and Characterization}

The chemical structure of GO and DGO are characterized by FTIR. As shown in Figure 2a, the stretching vibration of $\mathrm{O}-\mathrm{H}$ is signaled by the strong peak at $3400 \mathrm{~cm}^{-1}$. The peak at $1738 \mathrm{~cm}^{-1}$ can be ascribed to $\mathrm{C}=\mathrm{O}$ stretching of the $-\mathrm{COOH}$. The absorptions bands at 1629 and $1384-1051 \mathrm{~cm}^{-1}$ are attributed to $\mathrm{C}-\mathrm{O}-\mathrm{C} / \mathrm{C}-\mathrm{OH}$ stretching vibration and the remaining $\mathrm{sp}^{2}$ hybrid structure of $\mathrm{C}=\mathrm{C}$ [34]. Figure $2 \mathrm{~b}$ provides the FTIR spectrum of DGO. It can be clearly observed that the intensity of the peak at $1738 \mathrm{~cm}^{-1}$ is significantly reduced, probably due to the reaction of epoxy group of the GO with $-\mathrm{NH}_{2}$ of DAQ (see Figure 1). The 


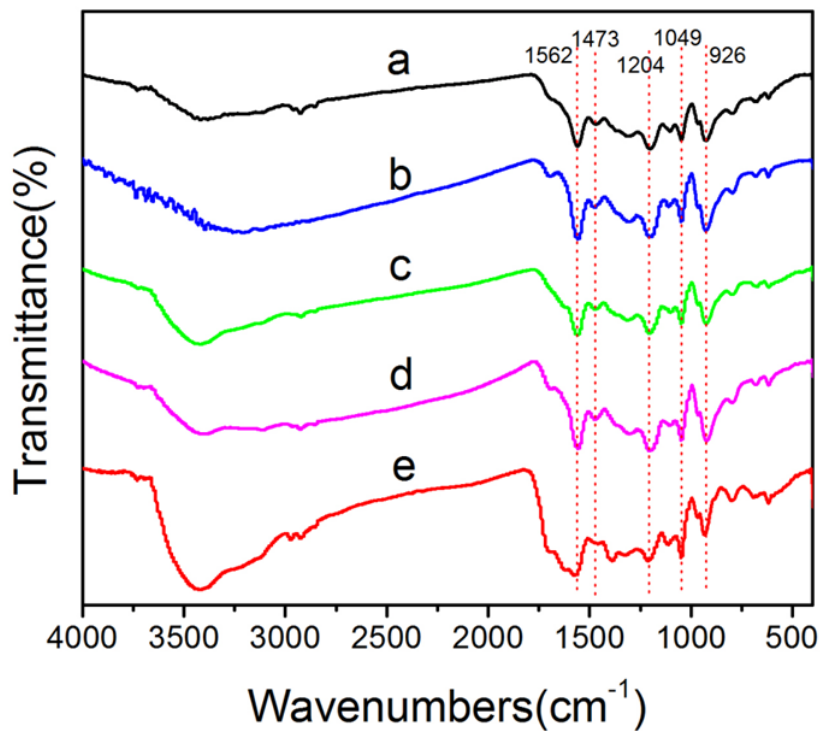

Figure 3. The FTIR spectra of (a) PPy, (b) DGP3, (c) DGP5, (d) DGP9 and (e) DGP15
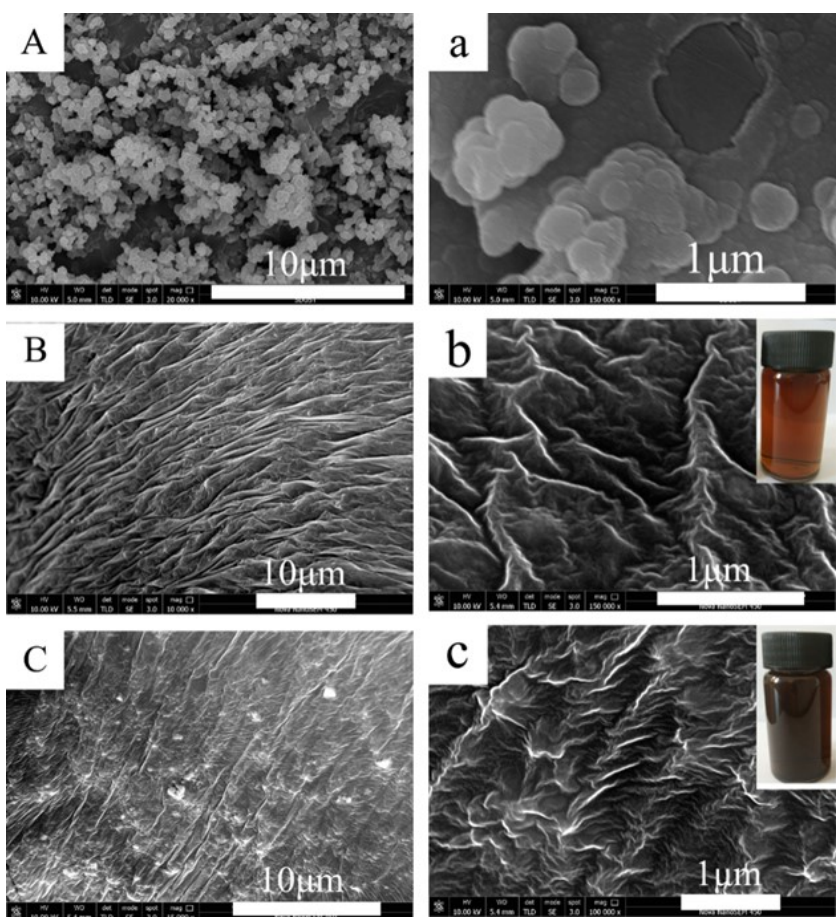

Figure 4. SEM images of (A,a) pure PPy, $(\mathrm{B}, \mathrm{b}) \mathrm{GO},(\mathrm{C}, \mathrm{c})$ DGO

in-plane bending vibration of $\mathrm{NH}-\mathrm{R}$ and stretching vibration of $\mathrm{C}-\mathrm{N}$ in $\mathrm{NH}-\mathrm{R}$ are also evidenced by the peaks located at $1575 \mathrm{~cm}^{-1}$ and 1271 $\mathrm{cm}^{-1}$, respectively. The above results indicate that DAQ is successfully grafted to the surface of GO.

Figure 3 shows the FTIR spectra of pure PPy and DGPs with different loadings. Typical absorption peaks of pure PPy can be found in Figure 3a. Peaks at 1562 and $1473 \mathrm{~cm}^{-1}$ are attributed to the stretching vibraion of pyrrole ring (symmetric) and pyrrole ring (asymmetric) [35]. The peak at 1204 and $1049 \mathrm{~cm}^{-1}$ are related to the in-plane vibration of $=\mathrm{C}-\mathrm{H}$ and the stretching vibration of $\mathrm{C}-\mathrm{N}$, respectively [36]. The peak at $926 \mathrm{~cm}^{-1}$ can be ascribed to the bipolaron state of PPy

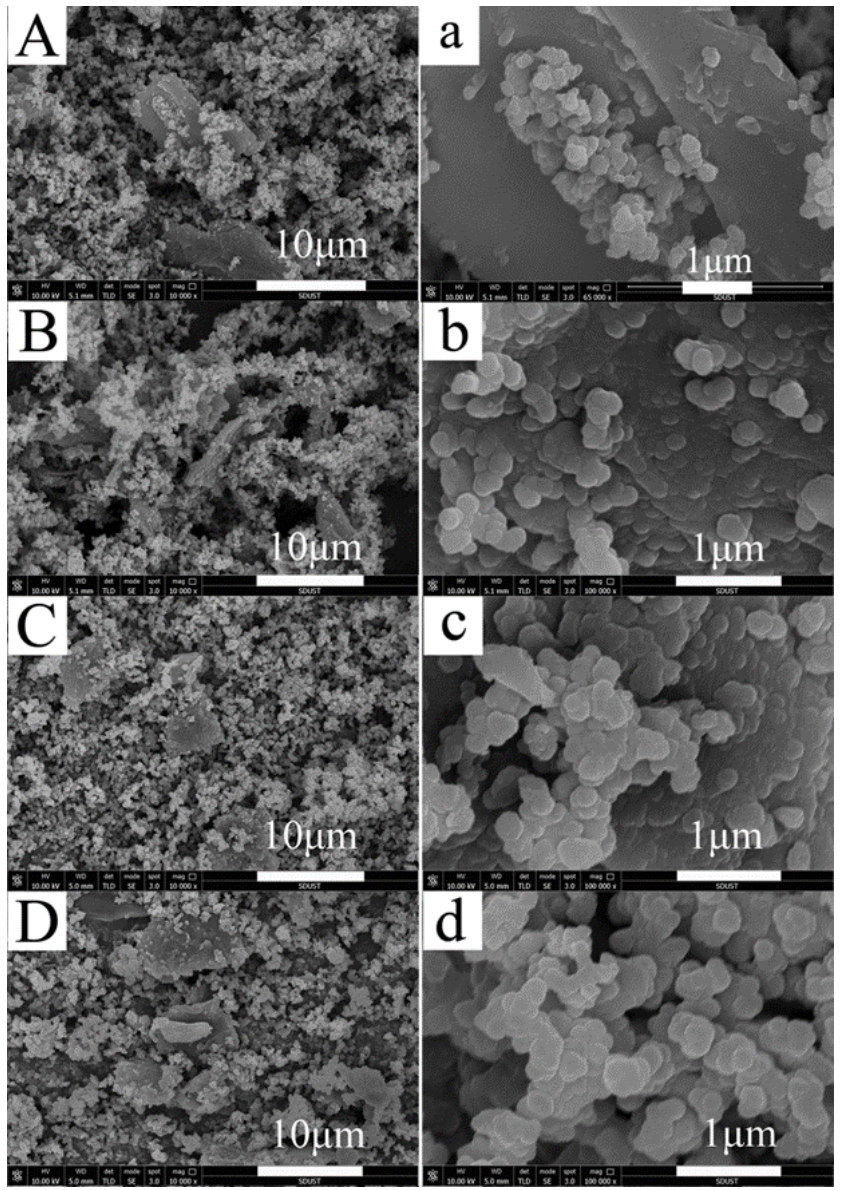

Figure 5. SEM images of (A)(a) DGP3, (B)(b) DGP5, (C)(c) DGP9 and (D)(d)DGP15

[37,38]. As shown in Figure 3b-3e, all DGPs provide characteristic peaks of PPy and there's no distinct peak shifts, which confirms that PPy is successfully polymerized in the presence of DGO and the chemical structure of PPy is not affected by the introduction of DGO.

Figure 4 provides the SEM images of pure PPy, GO and DGO at different magnifications. Pure PPy provides an irregular agglomerated granular morphology with 200-300nm in diameter (Figure 4a). Such agglomerated structure of the PPy is not favorable for supercapacitor applications since they minimize the surface area and have the tendency to block the access of electrolyte ions. As shown in Figure 4b, GO consists of randomly aggregated, thin and crumpled sheets. After reacted with DAQ, DGO provided a more rough morphology compared with pristine GO. More crumpled sheets can also be observed in Figure $4 \mathrm{c}$, which might be contributed to the graft reaction of DAQ with GO. Also being affected by the graft reaction, the color of DGO solutions changes from the light brown ( GO solution, see the top right corner of Figure $4 \mathrm{~b}$ ) to dark brown solution (see the top right corner of Figure 4c)

The surface morphologies of the composites are demonstrated in the SEM images of Figure 5. It can be clearly observed from Figure 5 that the morphologies of the DGPs are different from that of PPy. In the SEM image of DGP3 (Figure 5a), larger size of DGO sheets are sporadically distributed in PPy particles. When the DGO loading increases to $5 \mathrm{wt} \%$, smaller size of DGO sheets are embedded among the PPy particles in DGP5. It is noted that several DGO sheets bridge the PPy particles and thus form the interconnected microstructure, which would be in favor of the diffusion and migrations of the electrolyte ions when 


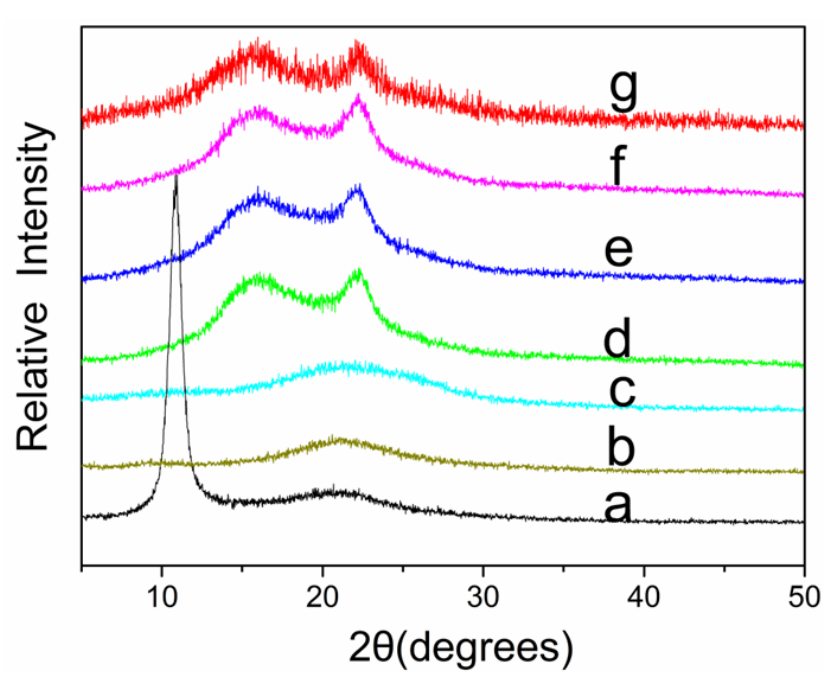

Figure 6. XRD spectra of (a) GO, (b) DGO (c) PPy, (d) DGP3, (e) DGP5, (f) DGP9 and (g)DGP15
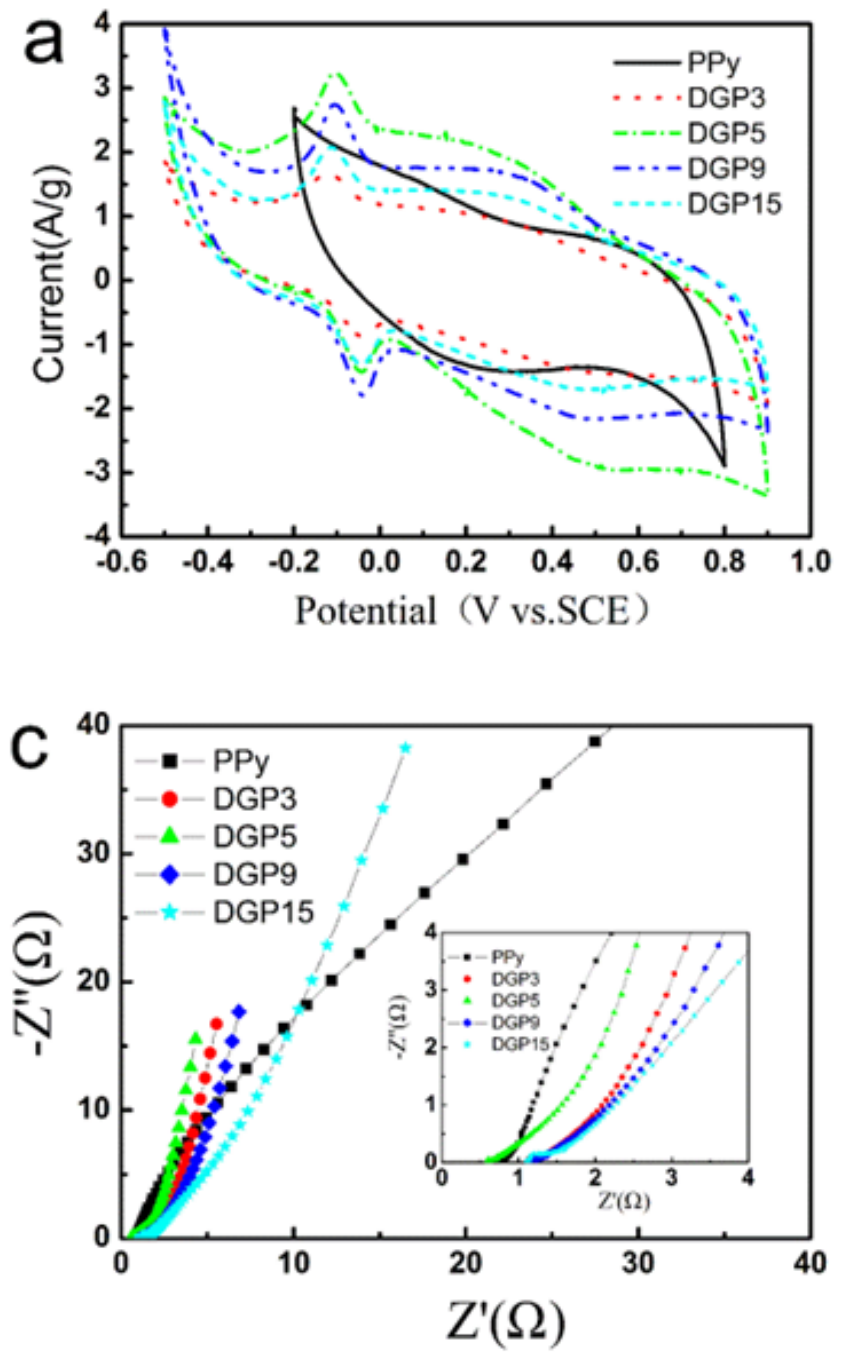

used as supercapacitor electrodes. As the DGO loading further increases, the interconnected microstructure cannot further be observed. However, stacking PPy particles are found in DGP9 and DGP15, which could have a negative effect on the further improvement of the electrochemical performances.

Figure 6 provides the XRD patterns of GO, DGO, PPy and different DGPs. The strong peak at $2 \theta=10.89^{\circ}$ in GO (Figure 6a) implies the successful synthesis of GO. It is noted that in the XRD pattern of DGO (Figure 6b) the characteristic peak of GO cannot further be detected, suggesting that the grafting process of GO might lead to the exfoliation of DGO sheets. A broad peak in the region of $2 \theta=20-30^{\circ}$ is observed in the spectrum of pristine PPy as shown in Figure 6c, indicating that the polymer is basically amorphous. Two diffraction peaks located at $2 \theta=15.8^{\circ}$ and $22.2^{\circ}$ can be found in DGP3, DGP5, DGP9 and DGP15, indicating that the oriented polymerization of PPy induced by the electronegative groups of DGO. The exfoliated DGO nanosheets embedded in the DGPs as well as the electroactivity of DGO might be benefit for the improvement of the electrochemical performances of DGPs.

\subsection{Electrochemical Performances}

CV, EIS, and GCD measurements are employed in a three-electrode system in order to study the electrochemical performances of the ob-
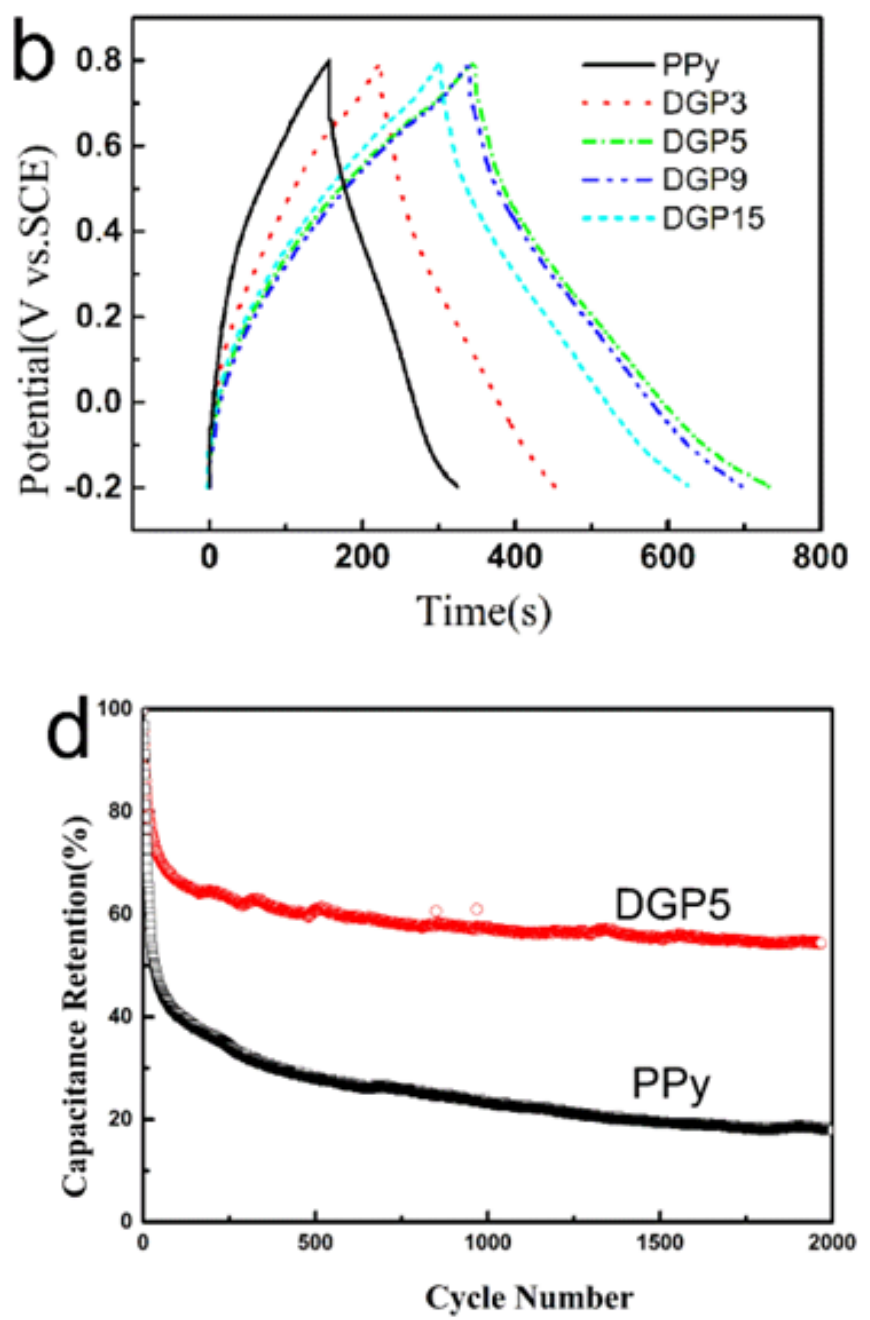

Figure 7. (a) $\mathrm{CV}$ curves at a scan rate of $10 \mathrm{mV} \mathrm{s}^{-1}$, (b) GCD curves at a current density of $1 \mathrm{~A} \mathrm{~g}^{-1}$, (c) Nyquist plots, (d) the specific capacitance change at a constant current density of $1 \mathrm{~A} \mathrm{~g} \mathrm{~g}^{-1}$ as a function of cycle number of the obtained samples 


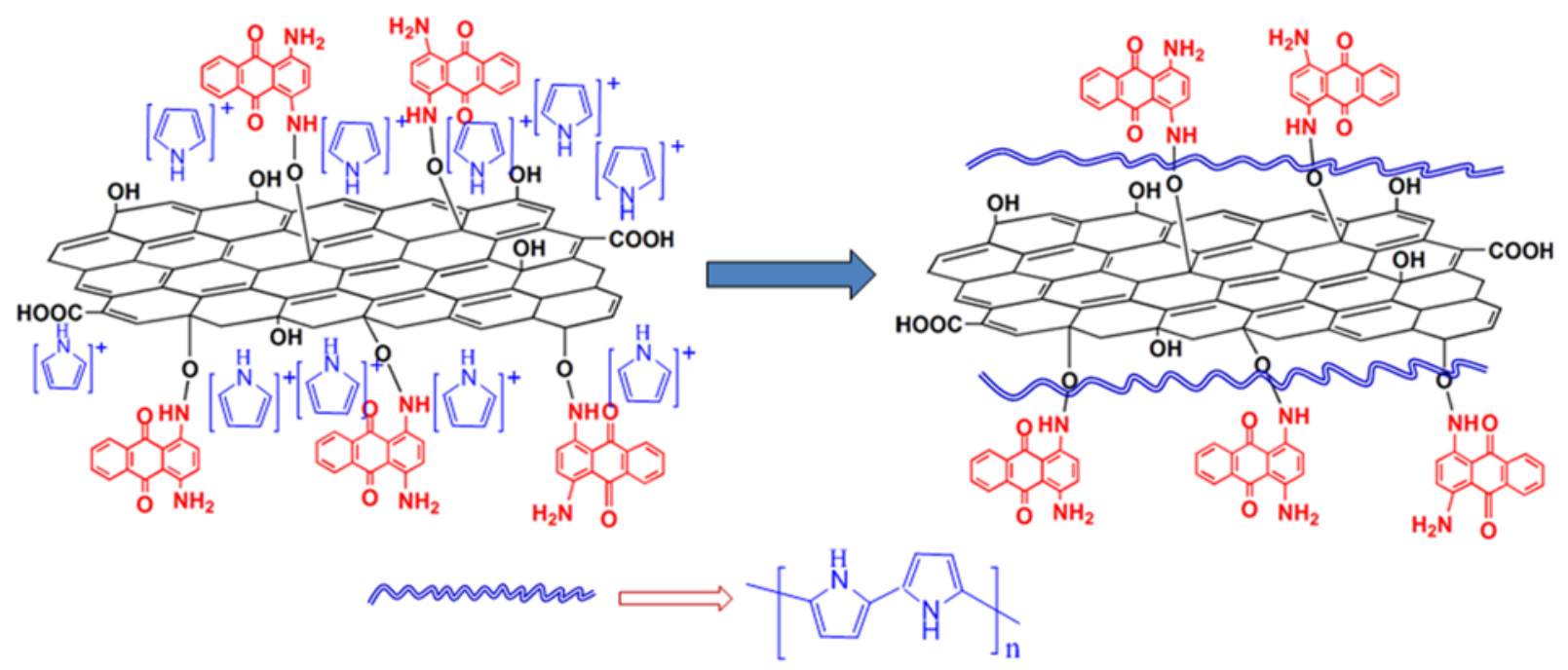

Figure 8. Scheme of the formation of DGP5

tained samples. Figure 7 shows a comparative study of the CV curves for PPy, DGP3, DGP5, DGP9 and DGP15 electrodes. As shown in Figure 7a the curve of PPy displays a rectangle-like shape, which exhibits a fast electrochemical switch. It can be clearly observed that the peak currents as well as the enclosed areas of DGPs are much larger than that of pristine PPy, indicating that the specific capacitances of DGPs are larger than that of PPy. It is worth noting that the potential window of DGPs $(-0.5 \mathrm{~V}$ to $0.9 \mathrm{~V})$ is enlarged compared with that of pure PPy $(-0.2 \mathrm{~V}-0.8 \mathrm{~V})$. The improved specific capacitance as well as the enlarged potential window might be contributed to the redox reaction of anthraquinone functional groups which is grafted onto DGO. A couple of redox peaks are also observed in the potential range of -0.2 to $0 \mathrm{~V}$, corresponding to the redox reaction of anthraquinone groups anchored on GO surface $[39,40]$. which is illustrated as follows.

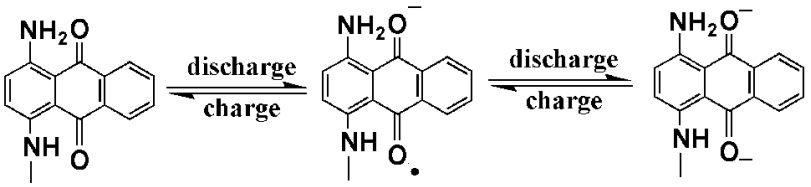

Moreover, nearly symmetrical shapes can be observed in the redox waves, suggesting good reversibility.

The specific capacitance of PPy and DGPs can be obtained by the GCD curves as shown in Figure $7 \mathrm{~b}$. In this paper, the current density we employed is $1 \mathrm{~A} \mathrm{~g} \mathrm{~g}^{-1}$. The specific capacitances of PPy, DGP3, DGP5, DGP9 and DGP15 electrode are 167, 224, 389, 367 and 325F g${ }^{1}$, respectively. The specific capacitances of DGPs are much larger than that of pristine PPy. The significant improved specific capacitance of DGP5 can be attributed to the redox-active DGO as well as the thus formed interconnected microstructure evidenced by SEM image. The interconnected microstructure can exhibit enhanced electrode/electrolyte interface areas, providing high electroactive regions and short diffusion lengths [41].

In order to further investigate the internal resistance, charge transfer and electrolyte diffusion process of the obtained samples. EIS measurement is carried out (Figure 7c). It can be seen that the resulting Nyquist plots comprise the semicircle arc at the high frequency region and the a straight line at the low frequency region. The semicircle at the high frequency region is originated from the charge transfer resistance (Rct), the value of which can be evaluated by the diameter of the semicircle. It can be seen from Figure 7c that the semicircle of the obtained sam- ples can hardly observed, indicating that the obtained samples all provide small charge transfer resistances. The internal resistance (Rs), evaluated from the intersection of the semicircle with $Z^{\prime}$ axis [42], is measured to be $\mathrm{t} 0.8,1.2,0.6,1.2$ and $1.2 \Omega$ for PPy, DGP3, DGP5, DGP9 and DGP15 electrode, respectively. The lower Rs of DGP5 than that of PPy and other DGPs could be ascribed to the interconnected conductive network which can favor electrons transfer efficiently. It can be seen that PPy provides a $45^{\circ}$ slope portion of curve, which is the Wurburge resistance, resulting from the frequency dependence of ion diffusion or transport. Compared with pristine PPy, DGP3, DGP5 and DGP9 show the nearly vertical line, indicating an ideal capacitive behavior and low diffusion resistance. Among the composites, DGP5 provides the best electrochemical performances. Therefore, this composite is selected for further investigations. The relationship between the specific capacitance and cycle number of DGP5 under the current density of $1 \mathrm{~A} \mathrm{~g}^{-1}$ in comparison with that of pure PPy is shown in Figure $7 \mathrm{~d}$. The capacitance retention of DGP5 is found higher (about 54\%) than that of pure PPy (only 18\%) after 2000 cycles at $1 \mathrm{~A} \mathrm{~g}^{-1}$, suggesting that the DGO could act as the mechanical support for PPy. Based on the above results, the introduction of DGO into PPy can promote the capacitance, stability and the cycle life of the electrodes.

It is worth noting that in DGP5, relatively smaller loading of DGO could play multiple roles in enhancing the electrochemical performances of PPy. Firstly, the DAQ electroactive molecules which are covalently grafted on the surface of GO might provide both the redox reactions contributing to the higher specific capacitance and the induction active sites for the polymerization of PPy due to its abundant electroactive groups and the aromatic chemical structure (Figure 8). Secondly, the small loading introduction of DGO can effectively hindered the accumulation of the PPy particles, and vice versa, the $\pi-\pi$ interactions as well as the formed hydrogen bond between PPy and DGO also prevents the stacking of DGO layers. Lastly, the delaminated DGO layers embedded in the loose PPy particles as a bridge and thus formed an interconnected porous microstructure, which might lead to more sites that are susceptible to redox reaction, a greater active area, consequently, higher capacitance.

\section{CONCLUSIONS}

In summary, DGO is polymerized by the ring opening reaction of epoxy group anchored on GO surface and $-\mathrm{NH}_{2}$ in DAQ. DGPs are polymerized by a facile chemical oxidative method with different DGO 
loadings $(3 w t \%, 5 w t \%, 9 w t \%$ and $15 w t \%)$. GO sheets are delaminated when grafted with DAQ, which is evidenced by X-ray diffractions. When the DGO loading reaches $5 \mathrm{wt} \%$, the delaminated sheets bridges the PPy particles and forms the interconnected microstructure. Compared with pristine PPy, the specific capacitances of DGPs are all improved and the potential window are enlarged due to the synergistic effects. Specifically, DGP5 provided both higher specific capacitances $\left(389 \mathrm{~F} \mathrm{~g}^{-1}\right)$ and better cycle stability than that of PPy. Moreover, working potential windows were enlarged to $1.4 \mathrm{~V}$ due to the redox activity of the DGO.

\section{ACKNOWLEDGEMENTS}

This work is supported by National Natural Science Foundation of China (Grant No. 51603116) . Yongqin Han is supported by China Scholarship Council (CSC).

\section{REFERENCES}

[1] Y.Y. Chen, M. Han, Y.J. Tang, J.C. Bao, S.L. Li, Y.Q. Lan, Z.H. Dai, Chem. Commun., 51, 12377 (2015).

[2] X.Q. Meng, H. Sun, J.W. Zhu, H.P. Bi, Q.F. Han, X.H. Liu, X. Wang, New J. Chem., 40, 2843(2016).

[3] M. Jiang, J.L. Zhang, F. Qiao, R.Y. Zhang, L.B. Xing, J. Zhou, H.Y. Cui, S.P. Zhuo, Rsc Adv., 6, 48276 (2016).

[4] L.B. Xing, J.L. Zhang, K. Qin, T.Z. Liu, J. Zhou,W.J. Si, S.P. Zhuo, Mater. Lett., 176, 189 (2016).

[5] J. Patiño, N. Lópezsalas, M.C. Gutiérrez, D. Carriazo, M.L. Ferrer, F.D. Monte, J. Mater. Chem. A, 4, 1251 (2016).

[6] T. Chen, R. Hao, H.S. Peng, L.M. Dai, Angew. Chem. Int. Ed., 54, 618 (2015).

[7] A. Sánchezsánchez, V. Fierro, M.T. Izquierdo, A. Celzard, J. Mater. Chem. A, 4, 6140 (2016).

[8] B. You, F. Kang, P.Q. Yin, Q. Zhang, Carbon,103, 9 (2016).

[9] R. Kötz, M. Carlen, Electrochim. Acta, 45, 2483 (2000).

[10]Z. Yu, L. Tetard, L. Zhai, J.Y. Thomas, Energ. Environ.Sci., 8 , 702 (2015)

[11]C.Z. Yuan, B. Gao, L.F. Shen, S.D. Yang, L. Hao, X.J. Lu, F. Zhang, L.J. Zhang, Nanoscale, 3,529 (2011).

[12]J. Yan, Q. Wang, C.P. Lin, T. Wei, Z.J. Fan, Adv. Energy Mater., 4, 1294 (2015).

[13]N.L. Torad, R.R. Salunkhe, Y. Li, H. Hamoudi, M. Imura, Y. Sakka, C.C. Hu, Y. Yamauchi, Chemistry, 20, 7895 (2014).

[14]A. Ramadoss, B. Saravanakumar, J.K. Sang, Nano Energy, 15,587(2015).

[15]H.J. Fei, C.Y. Yang, H. Bao, G.C. Wang, J. Power Sources, 266,488 (2014).

[16]Y.Y. Yang, Y.R. Liang, Y.D. Zhang, Z.Y. Zhang, Z.M. Li, Z.A. Hu, New J. Chem., 39, 4035, (2015).

[17]Y.H. Xue, L. Zhu, H. Chen, J. Qu, L.M. Dai, Carbon, 92,305 (2015).

[18]L. Mao, C. Guan, X.L. Huang, Q.Q. Ke, Y. Zhang, J. Wang, Electrochim. Acta, 196,653 (2016).

[19]C.L. Xiong, W.B. Zhong, Y.B. Zou, J.W. Luo, W.T. Yang, Electrochim. Acta , 211, 941 (2016).

[20]S. Ghasemi, S.R. Hosseini, P. Asen, Electrochim. Acta, 160,337 (2015).

[21]Y. Shi, L.J. Pan, B. Liu, Y.Q. Wang, Y. Cui, Z.N. Bao, G.H.Yu, J. Mater. Chem. A, 2,6086 (2014).

[22]Y.L. Chen, L.H. Du, P.H. Yang, P. Sun, X. Yu, W.J. Mai, J. Power Sources, 287,68 (2015) .

[23]C.H. Liu, Z.S. Cai, Y.P. Zhao, H. Zhao, F.Y. Ge, Cellulose, 23,
637 (2016).

[24]B. Gao, D. He, B. Yan, H. Suo, C. Zhao, J. Mater. Sci.: Mater. Electron., 26, 6373 (2015).

[25]J. Zhong, S. Gao, G.B. Xue, B. Wang, Macromolecules, 48, 1592 (2015).

[26]Y. Huang, H.F. Li, Z.F. Wang, M.S. Zhu, Z.X. Pei, Q. Xue, Y. Huang, C.Y. Zhi, Nano Energy, 22,422 (2016) .

[27]J.B. Zhu, Y.L. Xu, J.P. Wang, Y. Bai, X.F. Du, Phys. Chem. Chem. Phys., 17, 19885 (2015).

[28]Z.H. Chen, W.F. Liao, X.Y. Ni, Chem. Eng. J., 327, 1198 (2017).

[29]J. Li, Y. Zhang, H. Xie, Y. Li, C. Zhen, J. Solid State Electrochem., 21, 220 (2017).

[30]C. Yang, L. Zhang, N. Hu, Z. Yang, H. Wei, Y.Y. Wang, Y.F. Zhang, Appl. Surf. Sci., 387,666 (2016).

[31]Y.Q. Han, T.Q. Wang, T.X. Li, X.X. Gao, W. Li, Z.L. Zhang, Y.M. Wang, X.G. Zhang, Carbon, 119,111 (2017).

[32]J. Fernandez, J. Bonastre, J. Molina, F. Cases, European Polym. J., 103, 179(2018)

[33]L. Cao, F. Xu, Y.Y. Liang, H.L. Li, Adv. Mater., 16, 1853(2004).

[34]R. Bissesur, P.K.Y. Liu, W. White, S.F. Scully, Langmuir, 22, 1729 (2006).

[35]G.J. Cho, B.M. Fung, D.T. Glatzhofer, J.S. Lee, Y.G. Shu, Langmuir, 17, 456 (2001).

[36]E.J. Oh, K.S. Jang, A.G. MacDiarmid, Synth. Met., 125, 267 (2002).

[37]Y.C. Liu, Y.C. Liu, Y.T. Lin, J. Phys. Chem. B,107,11370 (2003).

[38]W.W. Chiu, J. Travaš-Sejdić, R.P. Cooney, G.A. Bowmaker, Synthetic Met., 155,80 (2005).

[39]Z. Algharaibeh, X.R.Liu, P.G. Pickup, J. Power Sources, 187,640 (2009).

[40]K. Kalinathan, D.P. DesRoches, X.R. Liu, P.G. Pickup, J. Power Sources, 181,182 (2008).

[41]H. Zhang, G.P. Cao, W.K. Wang, K.G. Yuan, B. Xu, W.F. Zhang, J. Cheng, Y.S. Yang, Electrochim. Acta ,54,1153 (2009).

[42]D. Kalpana, S.H. Cho, S.B. Lee, Y.S. Lee, R. Misra, N.G. Renganathan, J. Power Sources 190,587 (2009). 